Old Dominion University

ODU Digital Commons

Bioelectrics Publications

Frank Reidy Research Center for Bioelectrics

2014

\title{
Disassembly of Actin Structures by Nanosecond Pulsed Electric Field is a Downstream Effect of Cell Swelling
}

Andrei G. Pakhomov

Old Dominion University

Shu Xiao

Old Dominion University

Olga N. Pakhomova

Old Dominion University

Iurii Semenov

Old Dominion University

Marjorie A. Kuipers

See next page for additional authors

Follow this and additional works at: https://digitalcommons.odu.edu/bioelectrics_pubs

Part of the Biochemistry Commons, Biology Commons, Biophysics Commons, and the Other Life Sciences Commons

\section{Repository Citation}

Pakhomov, Andrei G.; Xiao, Shu; Pakhomova, Olga N.; Semenov, Iurii; Kuipers, Marjorie A.; and Ibey, Bennett L., "Disassembly of Actin Structures by Nanosecond Pulsed Electric Field is a Downstream Effect of Cell Swelling" (2014). Bioelectrics Publications. 183. https://digitalcommons.odu.edu/bioelectrics_pubs/183

\section{Original Publication Citation}

Pakhomov, A. G., Xiao, S., Pakhomova, O. N., Semenov, I., Kuipers, M. A., \& Ibey, B. L. (2014). Disassembly of actin structures by nanosecond pulsed electric field is a downstream effect of cell swelling. Bioelectrochemistry, 100, 88-95. doi:10.1016/

j.bioelechem.2014.01.004 
Authors

Andrei G. Pakhomov, Shu Xiao, Olga N. Pakhomova, Iurii Semenov, Marjorie A. Kuipers, and Bennett L. Ibey 


\title{
Disassembly of actin structures by nanosecond pulsed electric field is a downstream effect of cell swelling
}

\author{
Andrei G. Pakhomov ${ }^{a}$, Shu Xiao ${ }^{a, b}$, Olga N. Pakhomova ${ }^{a}$, lurii Semenov ${ }^{a}$, Marjorie A. \\ Kuipers $^{\mathrm{C}}$, and Bennett L. Ibey ${ }^{\mathrm{C}}$ \\ aFrank Reidy Research Center for Bioelectrics, Old Dominion University, Norfolk, VA, USA \\ bDept. of Electrical and Computer Engineering, Old Dominion University, Norfolk, VA, USA \\ 'Radio Frequency Bioeffects Branch, Bioeffects Division, Human Effectiveness Directorate, 711th \\ Human Performance Wing, Air Force Research Laboratory, Fort Sam Houston, TX, USA
}

\begin{abstract}
Disruption of the actin cytoskeleton structures was reported as one of the characteristic effects of nanosecond-duration pulsed electric field (nsPEF) in both mammalian and plant cells. We utilized $\mathrm{CHO}$ cells that expressed the monomeric fluorescent protein (mApple) tagged to actin to test if nsPEF modifies the cell actin directly or as a consequence of cell membrane permeabilization. A train of four 600-ns pulses at $19.2 \mathrm{kV} / \mathrm{cm}(2 \mathrm{~Hz})$ caused immediate cell membrane poration manifested by YO-PRO-1 dye uptake, gradual cell rounding and swelling. Concurrently, bright actin features were replaced by dimmer and uniform fluorescence of diffuse actin. To block the nsPEF-induced swelling, the bath buffer was isoosmotically supplemented with an electroporeimpermeable solute (sucrose). A similar addition of a smaller, electropore-permeable solute (adonitol) served as a control. We demonstrated that sucrose efficiently blocked disassembly of actin features by nsPEF, whereas adonitol did not. Sucrose also attenuated bleaching of mAppletagged actin in nsPEF-treated cells (as integrated over the cell volume), although did not fully prevent it. We conclude that disintegration of the actin cytoskeleton was a result of cell swelling, which, in turn, was caused by cell permeabilization by nsPEF and transmembrane diffusion of solutes which led to the osmotic imbalance.
\end{abstract}

\section{Keywords}

electric pulses; nanopores; plasma membrane; electroporation; cytoskeleton

\footnotetext{
(C) 2014 Elsevier B.V. All rights reserved.

Corresponding Author: Andrei G. Pakhomov, Frank Reidy Research Center for Bioelectrics, Old Dominion University, 4211 Monarch Way, Suite 300, Norfolk, VA 23508, 757-683-8003, 210-204-9012 (cell), 757-451-1010 (fax), 2andrei@ pakhomov.net.

Publisher's Disclaimer: This is a PDF file of an unedited manuscript that has been accepted for publication. As a service to our customers we are providing this early version of the manuscript. The manuscript will undergo copyediting, typesetting, and review of the resulting proof before it is published in its final citable form. Please note that during the production process errors may be discovered which could affect the content, and all legal disclaimers that apply to the journal pertain.
} 


\section{Introduction}

Permeabilization of cells and tissues by nanosecond pulsed electric field (nsPEF) is a recent extension of conventional electroporation technology [1-4]. A distinguishing feature of nsPEF is the ability to produce intracellular effects with relatively minimum disruption of the plasma membrane, resulting in a wide range of bioeffects. At the cellular level, such effects include the enhancement of transmembrane transport [5, 6], calcium mobilization [710], activation of phosphoinositide signaling [11] and MAPK pathways [12], phosphatidylserine externalization [13], mitochondrial membrane permeabilization [14], modulation of ion channels [15, 16], cell swelling and blebbing [17], and necrotic and apoptotic cell death $[1,18-21]$. In principle, intense nsPEF treatments are likely to modify most cell functions; however, the chain of events from the primary nsPEF effect of membrane permeabilization to secondary and further downstream effects is poorly understood. It is also being debated if permeabilization of membranous structures is the only primary effect of nsPEF. For example, a recent study established ROS formation by nsPEF in both living cells and culture media [22], although its biological significance has yet to be explored.

In recent years, specific attention has been paid to the disruption of actin microfilaments by nsPEF. This attention, at least in part, is fueled by profound differences in nsPEF sensitivity across different cell types $[18,23,24]$. Differences in the cytoskeleton organization and the robustness of the cytoskeletal cortex underlying the cell membrane could potentially explain the different vulnerability to electroporation. Indeed, in plant cells, stabilization of actin increased the stability of the plasma membrane against electric permeabilization as recorded by a decreased penetration of Trypan Blue into the cytoplasm [25]. In mammalian cells, inhibition of actin polymerization with Latrunculin A significantly enhanced cell uptake of propidium and phosphatidylserine externalization [26]. Nonetheless, the authors concluded that differences in the cell stiffness could only partially be responsible for cell vulnerability to nsPEF. In a different study, disruption of the actin cytoskeleton in adherent cells prior to nsPEF exposure significantly reduced cell survival [27], but it is not clear whether the combined effect was specific to nsPEF or just reflected general "weakening" of cells by the actin-disrupting toxin. Disintegration of the cytoskeleton in the cell cortex, followed by contraction of actin filaments towards the nucleus in cells irreversibly permeabilized by nsPEF [28] and degradation of actin cytoskeleton in cells undergoing nsPEF-induced apoptosis [29] established a further link between actin and the nsPEF-induced cell death. In contrast to the above observations, exposure of U937 cells to very long, high-rate nsPEF trains (e.g., 2400 pulses at $20 \mathrm{~Hz}, 60 \mathrm{~ns}, 10 \mathrm{kV} / \mathrm{cm}$ ) triggered rapid de novo actin cortex and scaffold formation within membrane blebs [30]; this phenomenon was observed only in a calcium-free bath buffer and led to the directional growth of giant longitudinal membrane protrusions capable of fast retraction after the exposure cessation.

It is now well established that intense nsPEF treatments permeabilize the cell plasma membrane to small solutes ("nanoelectroporation") [2, 5, 31-33]. These solutes travel down the electric and concentration gradients across the electroporated membrane, whereas larger solutes remain trapped inside the cell and create the osmotic force to attract water (the socalled colloid-osmotic mechanism) [17, 32, 34, 35]. The increased pressure from the inside 
forces the cell to acquire a more spherical shape and to use membrane reserves for cell volume increase. One can hypothesize that cell rounding and swelling are exactly the reasons for disassembly of actin cytoskeleton, in order to accommodate the new cell shape. On the other hand, one cannot exclude that it is the disintegration of the cytoskeleton by nsPEF that weakens the cell adhesion, makes cells more spherical, and allows for their swelling, which eventually culminates in membrane rupture and cell death. To resolve these two pathways, we blocked the colloid-osmotic swelling in nsPEF treated cells and demonstrated that it also prevents cell rounding and cytoskeleton disintegration.

\section{Materials and Methods}

\subsection{Cell line}

CHO-K1 cells (Chinese hamster ovary) were obtained from ATCC (Manassas, VA) and modified for stable expression of fluorescently tagged actin. The cells were transfected with a plasmid DNA encoding mApple-tagged human $\beta$-actin under a CMV promoter (a gift from Mike Davidson, Florida State University, Tallahassee, FL) using Effectene (Qiagen, Valencia, CA). Cells stably expressing mApple-actin were selected with $400 \mu \mathrm{g} / \mathrm{ml} \mathrm{G418}$ (Calbiochem, Darmstadt, Germany), screened for fluorescence using an inverted microscope, and isolated with cloning rings. The cells were propagated in $\mathrm{F} 12 \mathrm{~K}$ medium (Mediatech Cellgro, Herdon, VA) supplemented with 10\% fetal bovine serum (Atlanta Biologicals, Norcross, GA), 100 I.U./ml penicillin, $0.1 \mu \mathrm{g} / \mathrm{ml}$ streptomycin (Cellgro), and $400 \mu \mathrm{g} / \mathrm{ml} \mathrm{G} 418$. Cells were transferred onto poly-1-lysine covered glass coverslips 1-2 days prior to experiments. Transformed cells had the same appearance as the wild type $\mathrm{CHO}$ cells, but typically grew to a larger size.

\subsection{Cell imaging and data analysis}

A cover slip with cells was placed into a glass-bottomed chamber (Warner Instruments, Hamden, CT) mounted on an Olympus IX81 inverted microscope equipped with an FV 1000 confocal laser scanning system (Olympus America, Center Valley, PA). All experiments were performed at a room temperature of $22-24{ }^{\circ} \mathrm{C}$. Fluorescent images of tagged actin (excitation: $543 \mathrm{~nm}$; emission: 560-660 nm) were collected in parallel with differential-interference contrast (DIC) images using a 40x dry objective (NA 0.95). Although mApple emission is in the red spectrum, the fluorescent images were converted into a green pseudocolor for better clarity and visual contrast. For YO-PRO-1 dye uptake, we used a $488 \mathrm{~nm}$ laser and measured the emission in the $505-525 \mathrm{~nm}$ range.

In most experiments, we acquired either a time series of images of a selected X-Y plane, or $Z$-stacks of images at either 0.5 or $1 \mu \mathrm{m}$ distance between the planes. For the time series, the images were taken every $10 \mathrm{~s}$, and scanning for a single image took 5-7 s; three images were taken as a control prior to nsPEF delivery at $28 \mathrm{~s}$, and up to 40 images after nsPEF. The time intervals indicated below are those between the onset of nsPEF train and the onset of a particular image scan.

In contrast, Z-stacks of images were obtained at a higher resolution and scanning took long time (4-7 min). Therefore, in each experiment one Z-stack was collected immediately prior to nsPEF exposure and another one at $10 \mathrm{~min}$ after it (sometimes followed by a third one at 
$20 \mathrm{~min}$ ). 3D reconstruction of Z-stacks was accomplished with SlideBook 5.0 (Intelligent Imaging Innovations, Denver, $\mathrm{CO}$ ).

Images were quantified with MetaMorph Advanced v. 7.7.10.0 (Molecular Devices, Foster City, CA) and the numerical data were further processed with Grapher 9.6 (Golden Software, Golden, $\mathrm{CO}$ ). All numerical data in this paper are presented as mean values $+/-$ s.e. for 7-13 cells in each group (from a minimum of 3 independent experiments). The statistical significance of differences between individual groups was estimated with a twotailed $t$-test.

\subsection{Chemicals and buffers}

During experiments, the cells were kept in a physiological solution (hereinafter referred to as a "saline") containing (in $\mathrm{mM}$ ): $140 \mathrm{NaCl}, 5.4 \mathrm{KCl}, 1.5 \mathrm{MgCl}_{2}, 2 \mathrm{CaCl}_{2}, 10$ glucose, and

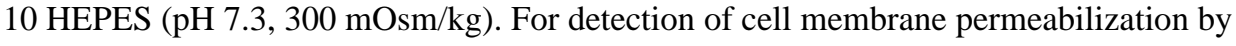
nsPEF, $1 \mu \mathrm{M}$ of YO-PRO-1 dye was added to the saline. In order to inhibit the nsPEFinduced cell swelling, the saline was mixed 7:3 with an isoosmotic water solution of sucrose. Since such modification of the saline also decreased its electrical conductance from 1.53 to $1.08 \mathrm{~S} / \mathrm{m}$ and it could change nsPEF effects, control samples were identically mixed with an isoosmotic adonitol solution. Saline with adonitol had the same conductance as saline with sucrose, but, due to smaller molecular size of adonitol, did not render protection against the colloid-osmotic swelling [17, 19].

Chemicals were purchased from Sigma-Aldrich (St. Louis, MO) except for YO-PRO-1 (Invitrogen, Eugene, OR).

\section{4. nsPEF exposure and dosimetry}

The method of nsPEF exposure was the same as described earlier [5, 6], with minor modifications. The electrode arrangement in relation to the cover slip and exposed cells was similar to shown in the previous papers [36, 37]. Nearly rectangular 600-ns pulses were generated in a transmission line-type circuit, by closing a MOSFET switch upon delivery of a TTL trigger pulse from pClamp software via a Digidata 1322A output (Molecular Devices). The same devices were employed to synchronize nsPEF delivery with image acquisition. NsPEF was delivered to a selected cell or a small group of cells on a glass coverslip with a pair of tungsten rod electrodes ( $100 \mu \mathrm{m}$ diameter) driven by a robotic manipulator (MP-225, Sutter, Novato, CA). The major difference from the previous studies was a narrower gap between the nsPEF-delivering electrodes $(100 \mu \mathrm{m})$ and a decreased height of the electrode tips above the coverslip $(30 \mu \mathrm{m})$. These changes were intended to maximize the E-field at the cell location, albeit at the expense of field uniformity. The nsPEF rise time (10 to $90 \%$ ) was $6.2 \mathrm{~ns}$.

The E-field at the cell location was determined by 3D simulations with a finite element Maxwell equations solver Amaze 3D (Field Precision, Albuquerque, NM). At the maximum charging voltage to the transmission line $(990 \mathrm{~V})$, the E- field at the cell location was 20 $\mathrm{kV} / \mathrm{cm}(+/-20 \%)$ for a single pulse and $19.2 \mathrm{kV} / \mathrm{cm}(+/-20 \%)$ for subsequent pulses when a train of pulses was delivered at $2 \mathrm{~Hz}$. This difference was caused by a limitation in pulser 
charging time. For clarity, the small difference between the first and next pulses in trains was disregarded, and in this paper we refer to pulse trains as being at $19.2 \mathrm{kV} / \mathrm{cm}$.

\section{Results and discussion}

\subsection{Permeabilization of cells with nsPEF and the choice of stimulation parameters}

In preliminary experiments, we attempted to modify the actin cytoskeleton by a single 600ns pulse at the amplitude of up to $20 \mathrm{kV} / \mathrm{cm}$. Except for the highest tested amplitude, the changes in cytoskeleton appearance were negligible and similar to "spontaneous" actin rearrangements continually going on in control cells (data not shown). To make sure that the pulses are properly delivered to cells and cause membrane permeabilization, the nsPEFinduced uptake of the YO-PRO-1 dye was employed as a positive control (Fig. 1). Indeed, a single pulse caused fast dye entry into the cells. While a single $20 \mathrm{kV} / \mathrm{cm}$ pulse was sufficient to cause actin rearrangements in some cells, in most experiments we chose to deliver brief trains of 4 pulses at $19.2 \mathrm{kV} / \mathrm{cm}$ which triggered a more reproducible and faster response. Of note, this treatment was more intense than the regimens that were utilized in other studies with $\mathrm{CHO}$ cells to drop the plasma membrane electrical resistance, to trigger $\mathrm{Tl}^{+}$and YO-PRO-1 uptake, and to activate phosphoinositide signaling $[5,11,38]$.

\section{2. nsPEF effects on actin features and on the cell shape}

Fig. 2 provides an example of the most profound changes that could be observed after a single $20 \mathrm{kV} / \mathrm{cm}$ pulse. Two shown Z-stacks of cell images were collected immediately before nsPEF (Fig. 2, A) and $20 \mathrm{~min}$ after it (Fig. 2, B). The exposure reduced the overall intensity of actin fluorescence, caused partial disassembly of filamentous actin structures and of non-filamentous actin "patches". The functional role of the non-filamentous actin structures is not fully understood (some of them could be focal adhesions); actin clusters, patches, or "clouds" were observed by other investigators, for example, during F-actin disintegration by latrunculin A [39] and before the formation of membrane blebs [40]. It is likely that the patches represent a transitional structure from the diffuse G-actin to highly organized actin filaments, and therefore contain partially polymerized protein. This would also explain why the patches appear more sensitive to nsPEF than highly organized actin stress fibers.

Concurrently, nsPEF-treated cells acquired a more spherical shape, expanding higher above the coverslip, and increased in volume. As discussed above, these changes resulted from water uptake due to the selective permeability of the electroporated membrane and the colloid-osmotic imbalance. In Fig. 3, the upper surface of the cell was scanned at $3 \mu \mathrm{m}$ above the coverslip surface before exposure (A), and expanded to $5.5 \mu \mathrm{m} 20 \mathrm{~min}$ later (B). These changes can be better appreciated from the side views of the cell obtained by a 3D reconstruction from Z-stacks (Fig. 2 C).

Exposure to 4 pulses caused even greater cell rounding and almost complete depolymerization of all actin structures (Fig. 3, B and C). The nsPEF-treated cells became almost half-spherical and were uniformly filled with diffuse actin (with the exception of the nucleus). Importantly, sham-exposed cells (zero pulses, but otherwise the same protocol) 
showed little or no changes in actin organization, no changes in the cell shape, and no bleaching (Fig. 3A).

Although water uptake is the driving force for cell volume change, it was not clear if rounding of the cell was a result of actin disintegration; or, vice versa, if the cell reshaping caused actin rearrangements. To answer this question, the following experiments were aimed to inhibit swelling and test if this would prevent the disassembly of actin features.

\subsection{Blockage of cell swelling helps to preserve actin structures}

The colloid-osmotic swelling is easy to prevent (or even revert into shrinking) by the addition of a pore-impermeable solute to the bath buffer while maintaining the overall osmolarity [17, 19]. For 600-ns pulses, the sucrose molecule was a sufficiently large and physiologically neutral solute that efficiently blocked swelling. The most convenient way to utilize sucrose for swelling prevention was pre-mixing of the saline with an isoosmotic sucrose solution, and the cells were placed into this mixture prior to nsPEF exposure (a replacement of the bath buffer after the exposure could shift the coverslip and alter the cell image).

A side effect of using the saline+sucrose buffer was a decreased electrical conductivity of the medium, which could potentially change the efficiency of electroporation [41]. To account for this effect, in control experiments we used an isoosmotic adonitol solution [17]. Adonitol is a smaller and also physiologically neutral sugar; it has the same effect as sucrose on the conductivity of the medium, but does not fully block the colloid-osmotic swelling (although markedly attenuates it as compared to the pure saline). The molar masses of adonitol and sucrose are 152 and $342 \mathrm{~g} / \mathrm{mol}$, respectively; their comparative structures and molecular dimensions were shown in our earlier study [17].

Fig. 4 compares changes in the actin organization and cell shape caused by nsPEF exposure in saline+sucrose (A and B) and in saline+adonitol (C and D). The cells exposed in the presence of sucrose displayed no swelling, and various actin features appeared wellpreserved. In contrast, adonitol only partially attenuated swelling (compared to pure saline in Fig. 3B) and failed to prevent actin bleaching and disintegration of actin filaments and patches.

\subsection{Quantitative analyses of the nsPEF effects}

For Fig. 5, the fluorescence of actin in each cell was integrated at each discrete height above the coverslip. After subtraction of noise (as measured in an area without cells), the postexposure emission intensity at each height was expressed in $\%$ to its pre-exposure level. The data were averaged for all treated cells and plotted separately for different bath buffer conditions. The sham-exposed cells showed just minor changes. Cells exposed to nsPEF in the saline as well as in the saline with adonitol displayed profound attenuation of emission in the lower layers above the coverslip along with its enhancement in the upper slices. This relocation of actin obviously reflects cell rounding and heightening above the coverslip.

Importantly, the graphs in Fig. 5 cannot be used to measure the cell height. The reason is the bleed-through of the fluorescence beyond the confocal plane, an inherent problem in the 
confocal imaging. Although the increased emission at higher levels indeed reflects the vertical expansion of the cell (and even more so considering overall bleaching, see Fig. 6B below), the vertical margin of the cell cannot be established from these measurements. The only legitimate conclusion from Fig. 5 is the upward migration of actin following nsPEF treatments when the cells were in the saline or in the saline+adonitol, but not in the saline +sucrose and not after a sham exposure.

Quantifying the extent of actin organization into various filament and non-filament structures was a challenge, primarily because of the great diversity of such structures within a cell and between different cells. The approach we used employed the Grid Measure function of MetaMorph. First, in the pre-exposure Z-stack we selected an X-Y slice which, just by sight, contained most of the structured actin. Next, a grid of 20 squares of $2-4 \mu^{2}$ was positioned over the area of best structured actin. The same area of the same slice was identified in the post exposure stack. If any of the grid squares appeared over the nucleus or beyond the cell margins, the grid position and/or size were adjusted. The mean fluorescence in each of 20 squares was automatically calculated. The standard deviation of the values measured in 20 grid squares was a fair reflection of whether actin was diffuse or structured, producing low and high values of the standard deviation, respectively. In order to account for possible bleaching, the standard deviation values were further normalized to the mean fluorescence intensity as averaged across the 20 grid squares. The resulting "granularity" index (where each grid square is counted as a granule) at 10 min post-exposure was expressed in $\%$ to the initial level in each cell, averaged for the cell population, and plotted in Fig. 6A.

Admittedly, measured granularity values varied substantially depending on the exact area where the grid was placed. Nonetheless, the averaged data portray correctly what was observed by a naked eye, namely a significant decay of actin structure when the nsPEFtreated cells were in the saline. The presence of extracellular sucrose prevented the decay, whereas adonitol failed to render the protection. These data clearly confirm that the nsPEFinduced disassembly of actin features was an effect downstream from cell swelling.

As noted above and can be seen from the images in Figs. 2-4 and graphs in Fig. 5, nsPEF decreased the actin fluorescence in the lower, actin-rich slices closer to the coverslip. To check if this was a consequence of the dye distribution over the larger volume of the swollen cell or a true bleaching effect, we have calculated the dye emission as integrated over the entire cell volume (Fig. 6B). In sham-exposed cells, the volume-integrated fluorescence did not diminish, thereby indicating that bleaching of the dye by repeated laser scanning was not the case. The integrated fluorescence was significantly decreased in all nsPEF-treated groups, although less so in the saline+sucrose. The generation and diffusion of reactive oxygen species is a common cause of bleaching of fluorescent dyes [42], and the recent observations of ROS generation by $n$ SPEF $[22,43]$ can provide a reasonable explanation to the observed bleaching of tagged actin. If this is indeed the case, then the protection from swelling rendered by sucrose has also reduced the intracellular ROS generation, e.g., by diminishing the damage to mitochondria (which would therefore also be a downstream consequence of swelling). This topic, however, goes beyond the scope of the present study. 


\subsection{Comparison with earlier studies and implications for future work}

Our observation that actin disassembly in mammalian CHO cells exposed to 600-ns pulses is a result of cell swelling appears contradictory to earlier findings in plant cells and using shorter $10 \mathrm{~ns}$ pulses $[25,28]$. These studies reported actin disassembly triggered by a single 10 -ns pulse at $33 \mathrm{kV} / \mathrm{cm}$, and the cell images taken before and after exposure show no evidence of cell swelling or reshaping. Of note, the exposure buffer for plant cells contained a significant amount of sorbitol (not clear if it was a standard constituent of the medium for plant cells or was deliberately added to block swelling). There is no universally accepted approach to compare the efficiency of 10- and 600-ns pulses; however, all published comparison methods $[18,38,44,45]$ indicate that a single 10 -ns pulse at $33 \mathrm{kV} / \mathrm{cm}$ is a much milder treatment than a train of four $600-\mathrm{ns}$ pulses at $19.2 \mathrm{kV} / \mathrm{cm}$. Thus, the relatively mild treatment with 10-ns pulses caused no cell swelling (either because of sorbitol or because of relatively minor membrane disruption) but nonetheless caused actin disassembly. Physiological differences between plant and mammalian cells may in part be responsible for this difference, but the data are also indicative of some qualitatively different effect of 10-ns pulses. This conclusion would also be consistent with a much higher efficiency of $10 \mathrm{~ns}$ pulses to mobilize calcium from the endoplasmic reticulum, which was interpreted as a manifestation of the intracellular electroporation [9]. A study that compares the effects of 10- and 600-ns pulses on actin under similar conditions and in the same object would be most useful to determine if the difference is indeed caused by the direct intracellular effect of 10-ns pulses.

\section{Conclusion}

We found that an intense exposure of mammalian CHO cells to $600 \mathrm{~ns}$ pulses caused disassembly of the organized actin. This effect was a downstream consequence of the nsPEF-induced cell swelling and could be prevented by the inhibition of swelling with extracellular sucrose. These data are contradictory to earlier findings with 10-ns pulses in plant cells and may be indicative of a qualitative difference between the effects of 10- and 600-ns electric pulses.

\section{Acknowledgments}

The study was supported by R01CA125482 from the National Cancer Institute and R01GM088303 from the National Institute of General Medical Sciences (AGP), and by the Air Force Office of Scientific Research LRIR 13RH08COR (BLI).

\section{Abbreviations}

$\begin{array}{ll}\text { DIC } & \text { differential interference contrast } \\ \text { nsPEF } & \text { nanosecond pulsed electric field } \\ \text { ROS } & \text { reactive oxygen species }\end{array}$

\section{References}

1. Schoenbach KS, Hargrave B, Joshi RP, Kolb J, Osgood C, Nuccitelli R, Pakhomov AG, Swanson J, Stacey M, White JA, Xiao S, Zhang J, Beebe SJ, Blackmore PF, Buescher ES. Bioelectric Effects of 
Nanosecond Pulses. IEEE Transactions on Dielectrics and Electrical Insulation. 2007; 14:10881109.

2. Pakhomov, AG.; Miklavcic, D.; Markov, MS., editors. Advanced Electroporation Techniques in Biology in Medicine. CRC Press; Boca Raton: 2010. p. 528

3. Breton M, Mir LM. Microsecond and nanosecond electric pulses in cancer treatments. Bioelectromagnetics. 2012; 33:106-123.

4. Schoenbach KH, Beebe SJ, Buescher ES. Intracellular effect of ultrashort electrical pulses. Bioelectromagnetics. 2001; 22:440-448. [PubMed: 11536285]

5. Bowman AM, Nesin OM, Pakhomova ON, Pakhomov AG. Analysis of plasma membrane integrity by fluorescent detection of $\mathrm{Tl}^{+}$uptake. J Membr Biol. 2010; 236:15-26. [PubMed: 20623351]

6. Pakhomov AG, Bowman AM, Ibey BL, Andre FM, Pakhomova ON, Schoenbach KH. Lipid nanopores can form a stable, ion channel-like conduction pathway in cell membrane. Biochem Biophys Res Commun. 2009; 385:181-186. [PubMed: 19450553]

7. Vernier PT, Sun Y, Chen MT, Gundersen MA, Craviso GL. Nanosecond electric pulse-induced calcium entry into chromaffin cells. Bioelectrochemistry. 2008; 73:1-4. [PubMed: 18407807]

8. Craviso GL, Choe S, Chatterjee P, Chatterjee I, Vernier PT. Nanosecond electric pulses: a novel stimulus for triggering $\mathrm{Ca}^{2+}$ influx into chromaffin cells via voltage-gated $\mathrm{Ca}^{2+}$ channels. Cell Mol Neurobiol. 2010; 30:1259-1265. [PubMed: 21080060]

9. Semenov I, Xiao S, Pakhomova ON, Pakhomov AG. Recruitment of the intracellular $\mathrm{Ca}^{2+}$ by ultrashort electric stimuli: The impact of pulse duration. Cell Calcium. 2013; 15:00083-00083.

10. Semenov I, Xiao S, Pakhomov AG. Primary pathways of intracellular $\mathrm{Ca}^{2+}$ mobilization by nanosecond pulsed electric field. Biochim Biophys Acta. 2013; 1828:981-989. [PubMed: 23220180]

11. Tolstykh GP, Beier HT, Roth CC, Thompson GL, Payne JA, Kuipers MA, Ibey BL. Activation of intracellular phosphoinositide signaling after a single 600 nanosecond electric pulse. Bioelectrochemistry. 2013; 94C:23-29. [PubMed: 23747521]

12. Morotomi-Yano K, Akiyama H, Yano K. Nanosecond pulsed electric fields activate MAPK pathways in human cells. Archives of Biochemistry and Biophysics. 2011; 515:99-106. [PubMed: 21933660]

13. Vernier PT, Ziegler MJ, Sun Y, Gundersen MA, Tieleman DP. Nanopore-facilitated, voltagedriven phosphatidylserine translocation in lipid bilayers--in cells and in silico. Phys Biol. 2006; 3:233-247. [PubMed: 17200599]

14. Napotnik TB, Wu YH, Gundersen MA, Miklavcic D, Vernier PT. Nanosecond electric pulses cause mitochondrial membrane permeabilization in Jurkat cells. Bioelectromagnetics. 2012; 33:257-264. [PubMed: 21953203]

15. Nesin V, Pakhomov AG. Inhibition of voltage-gated $\mathrm{Na}(+)$ current by nanosecond pulsed electric field (nsPEF) is not mediated by $\mathrm{Na}^{+}$influx or $\mathrm{Ca}^{2+}$ signaling. Bioelectromagnetics. 2012; 33:443-451. [PubMed: 22234846]

16. Nesin V, Bowman AM, Xiao S, Pakhomov AG. Cell permeabilization and inhibition of voltagegated $\mathrm{Ca}^{2+}$ and $\mathrm{Na}^{+}$channel currents by nanosecond pulsed electric field. Bioelectromagnetics. 2012; 33:394-404. [PubMed: 22213081]

17. Nesin OM, Pakhomova ON, Xiao S, Pakhomov AG. Manipulation of cell volume and membrane pore comparison following single cell permeabilization with 60- and 600-ns electric pulses. Biochim Biophys Acta. 2011; 1808:792-801. [PubMed: 21182825]

18. Ibey BL, Pakhomov AG, Gregory BW, Khorokhorina VA, Roth CC, Rassokhin MA, Bernhard JA, Wilmink GJ, Pakhomova ON. Selective cytotoxicity of intense nanosecond-duration electric pulses in mammalian cells. Biochim Biophys Acta. 2010; 1800:1210-1219. [PubMed: 20691249]

19. Pakhomova ON, Gregory BW, Semenov I, Pakhomov AG. Two modes of cell death caused by exposure to nanosecond pulsed electric field. PLoS One. 2013; 8:e70278. [PubMed: 23894630]

20. Beebe SJ, Fox PM, Rec LJ, Willis EL, Schoenbach KH. Nanosecond, high-intensity pulsed electric fields induce apoptosis in human cells. Faseb J. 2003; 17:1493-1495. [PubMed: 12824299]

21. Ren W, Beebe SJ. An apoptosis targeted stimulus with nanosecond pulsed electric fields (nsPEFs) in E4 squamous cell carcinoma. Apoptosis. 2011; 16:382-393. [PubMed: 21213047] 
22. Pakhomova ON, Khorokhorina VA, Bowman AM, Rodaite-Riseviciene R, Saulis G, Xiao S, Pakhomov AG. Oxidative effects of nanosecond pulsed electric field exposure in cells and cellfree media. Arch Biochem Biophys. 2012; 527:55-64. [PubMed: 22910297]

23. Yang W, Wu YH, Yin D, Koeffler HP, Sawcer DE, Vernier PT, Gundersen MA. Differential sensitivities of malignant and normal skin cells to nanosecond pulsed electric fields. Technol Cancer Res Treat. 2011; 10:281-286. [PubMed: 21517135]

24. Ibey BL, Roth CC, Pakhomov AG, Bernhard JA, Wilmink GJ, Pakhomova ON. Dose-dependent thresholds of 10-ns electric pulse induced plasma membrane disruption and cytotoxicity in multiple cell lines. PLoS One. 2011; 6:e15642. [PubMed: 21390200]

25. Hohenberger P, Eing C, Straessner R, Durst S, Frey W, Nick P. Plant actin controls membrane permeability. Biochim Biophys Acta. 2011; 1808:2304-2312. [PubMed: 21669183]

26. Thompson GL, Roth C, Tolstykh G, Kuipers M, Ibey BL. Role of cytoskeleton and elastic moduli in cellular response to nanosecond pulsed electric fields. Proc SPIE 8585, Terahertz and Ultrashort Electromagnetic Pulses for Biomedical Applications, 85850T. Feb 23.2013

27. Stacey M, Fox P, Buescher S, Kolb J. Nanosecond pulsed electric field induced cytoskeleton, nuclear membrane and telomere damage adversely impact cell survival. Bioelectrochemistry. 2011; 82:131-134. [PubMed: 21719360]

28. Berghofer T, Eing C, Flickinger B, Hohenberger P, Wegner LH, Frey W, Nick P. Nanosecond electric pulses trigger actin responses in plant cells. Biochem Biophys Res Commun. 2009; 387:590-595. [PubMed: 19619510]

29. Ford WE, Ren W, Blackmore PF, Schoenbach KH, Beebe SJ. Nanosecond pulsed electric fields stimulate apoptosis without release of pro-apoptotic factors from mitochondria in B16f10 melanoma. Archives of Biochemistry and Biophysics. 2010; 497:82-89. [PubMed: 20346344]

30. Rassokhin MA, Pakhomov AG. Electric field exposure triggers and guides formation of pseudopod-like blebs in U937 monocytes. J Membr Biol. 2012; 245:521-529. [PubMed: 22638933]

31. Vernier PT, Sun Y, Gundersen MA. Nanoelectropulse-driven membrane perturbation and small molecule permeabilization. BMC Cell Biol. 2006; 7:37. [PubMed: 17052354]

32. Pakhomov, AG.; Pakhomova, ON. Nanopores: A distinct transmembrane passageway in electroporated cells. In: Pakhomov, AG.; Miklavcic, D.; Markov, MS., editors. Advanced Electroporation Techniques in Biology in Medicine. CRC Press; Boca Raton: 2010. p. 178-194.

33. Silve A, Leray I, Mir LM. Demonstration of cell membrane permeabilization to medium-sized molecules caused by a single 10 ns electric pulse. Bioelectrochemistry. 2012; 87:260-264. [PubMed: 22074790]

34. Kinosita K Jr, Tsong TY. Voltage-induced pore formation and hemolysis of human erythrocytes. Biochim Biophys Acta. 1977; 471:227-242. [PubMed: 921980]

35. Serpersu EH, Kinosita K Jr, Tsong TY. Reversible and irreversible modification of erythrocyte membrane permeability by electric field. Biochim Biophys Acta. 1985; 812:779-785. [PubMed: 3970906]

36. Roth CC, Tolstykh GP, Payne JA, Kuipers MA, Thompson GL, DeSilva MN, Ibey BL. Nanosecond pulsed electric field thresholds for nanopore formation in neural cells. Journal of biomedical optics. 2013; 18:035005. [PubMed: 23532338]

37. Pakhomov AG, Kolb JF, White JA, Joshi RP, Xiao S, Schoenbach KH. Long-lasting plasma membrane permeabilization in mammalian cells by nanosecond pulsed electric field (nsPEF). Bioelectromagnetics. 2007; 28:655-663. [PubMed: 17654532]

38. Ibey BL, Xiao S, Schoenbach KH, Murphy MR, Pakhomov AG. Plasma membrane permeabilization by 60 - and 600 -ns electric pulses is determined by the absorbed dose. Bioelectromagnetics. 2009; 30:92-99. [PubMed: 18839412]

39. Gerisch G, Bretschneider T, Muller-Taubenberger A, Simmeth E, Ecke M, Diez S, Anderson K. Mobile actin clusters and traveling waves in cells recovering from actin depolymerization. Biophys J. 2004; 87:3493-3503. [PubMed: 15347592]

40. Ballestrem C, Wehrle-Haller B, Imhof BA. Actin dynamics in living mammalian cells. J Cell Science. 1998; 111(Pt 12):1649-1658. [PubMed: 9601095] 
41. Sukhorukov VL, Mussauer H, Zimmermann U. The effect of electrical deformation forces on the electropermeabilization of erythrocyte membranes in low- and high-conductivity media. J Membr Biol. 1998; 163:235-245. [PubMed: 9625780]

42. Spence, MTZ; Johnson, ID. The molecular probes handbook: a guide to fluorescent probes and labeling technologies. 11. Live Technologies Corporation; Carlsbad, CA: 2010.

43. Nuccitelli R, Lui K, Kreis M, Athos B, Nuccitelli P. Nanosecond pulsed electric field stimulation of reactive oxygen species in human pancreatic cancer cells is $\mathrm{Ca}^{2+}$-dependent. Biochem Biophys Res Commun. 2013; 435:580-585. [PubMed: 23680664]

44. Ibey BL, Mixon DG, Payne JA, Bowman A, Sickendick K, Wilmink GJ, Roach WP, Pakhomov AG. Plasma membrane permeabilization by trains of ultrashort electric pulses. Bioelectrochemistry. 2010; 79:114-121. [PubMed: 20171148]

45. Schoenbach K, Joshi R, Beebe S, Baum C. A scaling law for membrane permeabilization with nanopulses. IEEE Transactions on Dielectrics and Electrical Insulation. 2009; 16:1224-1235.

\section{Biographies}

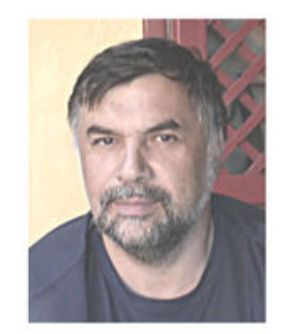

Andrei G. Pakhomov received his M.S. degree in animal and human physiology from Moscow State University in 1982, and a Ph.D. in radiation biology from the Medical Radiology Research Center in Obninsk, Russia, in 1989. He is a Research Professor with Frank Reidy Research Center for Bioelectrics of Old Dominion University in Norfolk, VA. His research is focused on cell membrane permeabilization by nanosecond-duration electric pulses, including biophysical mechanisms of pore formation and resealing, nanopore conductive properties, physiological consequences of nanoelectroporation, and its applications in biology and medicine.

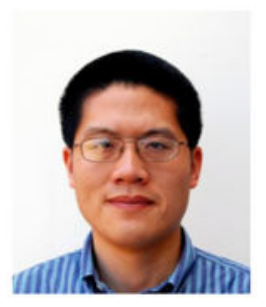

Shu Xiao received the Ph.D. degree in electrical engineering from Old Dominion University, Norfolk, VA, in 2004. He is currently an Assistant Professor at the Department of Electrical and Computer Engineering, Old Dominion University, teaching power electronics and electric drives. He is also working at the Frank Reidy Research Center for Bioelectrics. His research interests include pulsed power, high power electromagnetics, electrical discharges in liquids and gases. His research interests also include using pulsed power and pulsed electromagnetic radiation in therapeutics and imaging applications. 


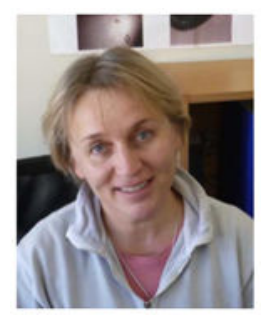

Olga N. Pakhomova is a Research Associate Professor at Old Dominion University in the Frank Reidy Research Center for Bioelectric. She received her Ph.D. from the Medical Radiology Research Center (Russia) and did postdoctoral training at University of Texas Health Science Center at San Antonio. Her research is centered on understanding and characterization of cell response to treatment with electric pulses, with emphasis on nanosecond-duration pulses and biophysical properties of membrane nanopores. Main topics are the escalation of cell sensitivity to pulsed electric field (PEF) for advancement of PEFmediated tissue and tumor ablation, and mechanisms of the PEF-triggered cell death.

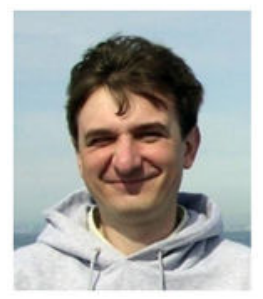

Iurii Semenov received a M.S. (1996) and Ph.D. (2002) degrees in biophysics at Taras Shevchenko Kyiv National University in Ukraine. He is Research Assistant Professor at Frank Reidy Research Center for Bioelectrics. His current research interests are focused on intracellular effects of nanosecond pulsed electric fields; molecular mechanisms of intracellular calcium regulation and signal-transduction pathways in excitable and nonexcitable cells.

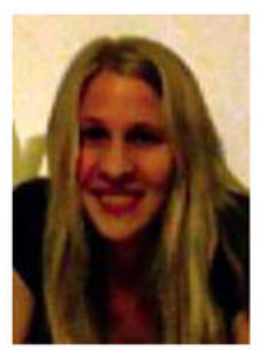

Marjorie Ann Kuipers received a BS in Cellular \& Molecular Biology from the University of Michigan in 2002, an MS in toxicology from the University of Florida in 2007, and a PhD in biology from Florida State University in 2010. She is currently pursuing a DVM from the University of Florida with a public health interest in animal and agricultural production in the China and Persian Gulf regions. 


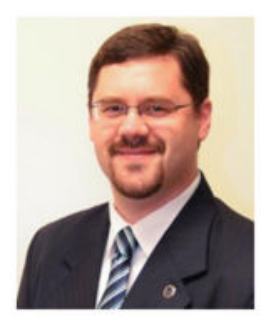

Bennett L. Ibey is a Senior Research Biomedical Engineer with the 711 Human Performance Wing of the Air Force Research Laboratory, Radio Frequency Bioeffects Branch. He received a Ph.D. in Biomedical Engineering from Texas A\&M University in 2006 with a focus on biomedical optics. Since that time, his research interests have focused on the interaction between tissue and nanosecond pulsed electric fields and high peak power microwave bioeffects. 


\section{Highlights}

- Nanosecond pulsed electric field (nsPEF) causes disassembly of actin structures

- Same nsPEF exposure triggers cell swelling and reshaping

- Swelling and reshaping are blocked by the extracellular sucrose but not by adonitol

- Sucrose but not adonitol also inhibited nsPEF-induced disassembly of actin features 

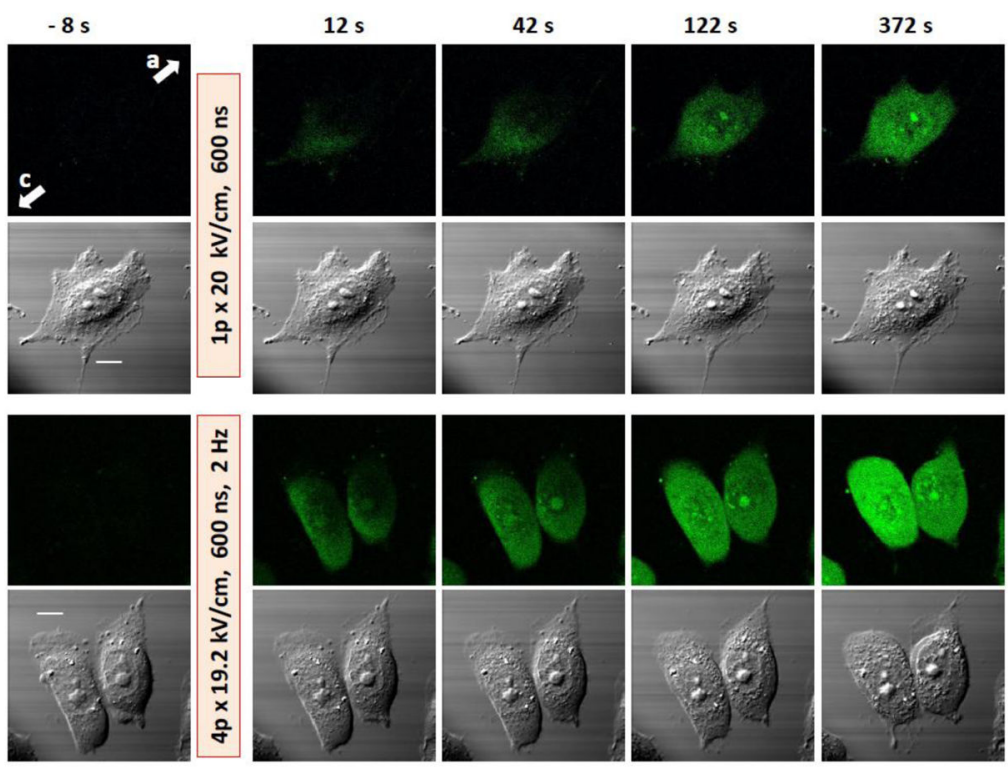

Fig. 1.

Permeabilization of CHO cells by 600 ns pulses as evidenced by Yo-PRO-1 dye uptake. Shown are representative frames of a time series of fluorescence images (top rows) and simultaneous DIC images taken before nsPEF (left, $-8 \mathrm{~s}$ ) and at different intervals after it. The exposure parameters are indicated in the figure. Arrows with letters "c" and "a" show the directions to the cathode and anode nsPEF delivering electrodes, respectively (applies to all images). The scale bar is $10 \mu \mathrm{m}$. Note gradual dye entry from the cathode-facing pole of the cell and the more profound effect of the 4-pulse train. Changes in DIC images were modest with 1 pulse but obvious after 4 pulses (cell rounding, granulation, and retraction of lamillipodia). 
A

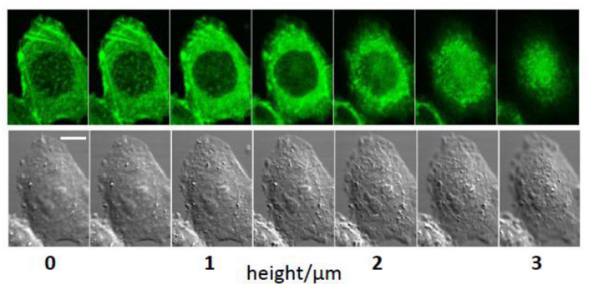

B

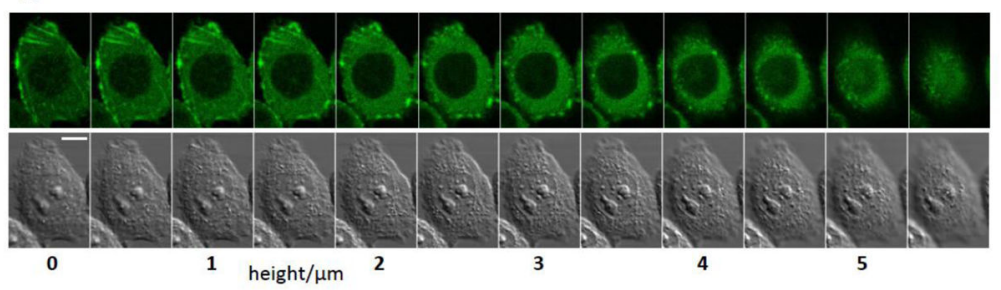

Fig. 2.

Cell swelling and reorganization of actin following nsPEF exposure (1 pulse at $20 \mathrm{kV} / \mathrm{cm}$, $600 \mathrm{~ns})$. A and B: Z-stacks of fluorescence and DIC images of the same cell taken immediately prior to nsPEF (A) and 20 min after it (B). The leftmost images are at the level of the coverslip surface (the bottom of the cell), and the height is increased in $0.5 \mu \mathrm{m}$ steps. $\mathrm{C}$ : side views of the cell actin reconstructed from Z-stacks in A (top, before exposure) and B (bottom, 20 min post nsPEF). The scale bars are $10 \mu \mathrm{m}$ (A and B) and $5 \mu \mathrm{m}$ (C). Note cell swelling, partial disassembly of actin features, and overall reduction of fluorescence after nsPEF. 


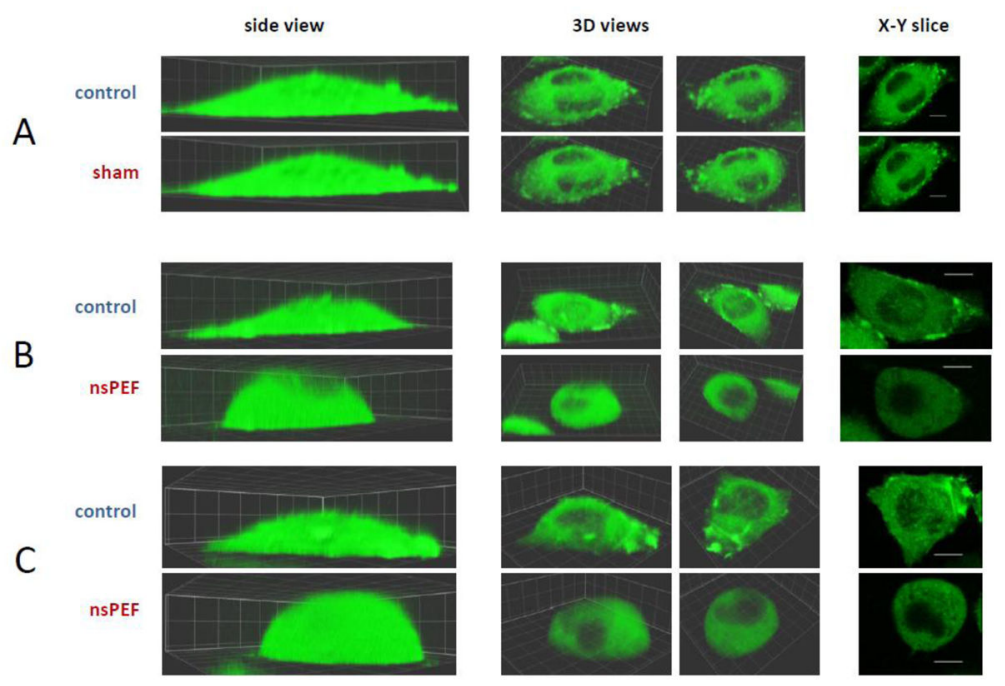

Fig. 3.

Cell reshaping and disassembly of structured actin by nsPEF. A, B, and C are three representative experiments (out of 18). Identical Z-stacks of cell images were taken immediately before exposure (top rows, "control") and $10 \mathrm{~min}$ after it (bottom rows). Cells were exposed to either 0 pulses in A (sham exposure) or 4 pulses at $19.2 \mathrm{kV} / \mathrm{cm}(600 \mathrm{~ns}, 2$ $\mathrm{Hz}$ ) in B and $\mathrm{C}$. The side views (left column) are intended to demonstrate cell reshaping by nsPEF. Two different 3D views (center columns) and one X-Y slice (right) were selected to provide the best observation of the structured actin. For each experiment, the control and post-nsPEF images show the same 3D views and the same X-Y slice. For the side and 3D views, the grid size is 5 x $5 \mu \mathrm{m}$; for slices, the bar is $10 \mu \mathrm{m}$. All experiments were performed in the physiological saline. 


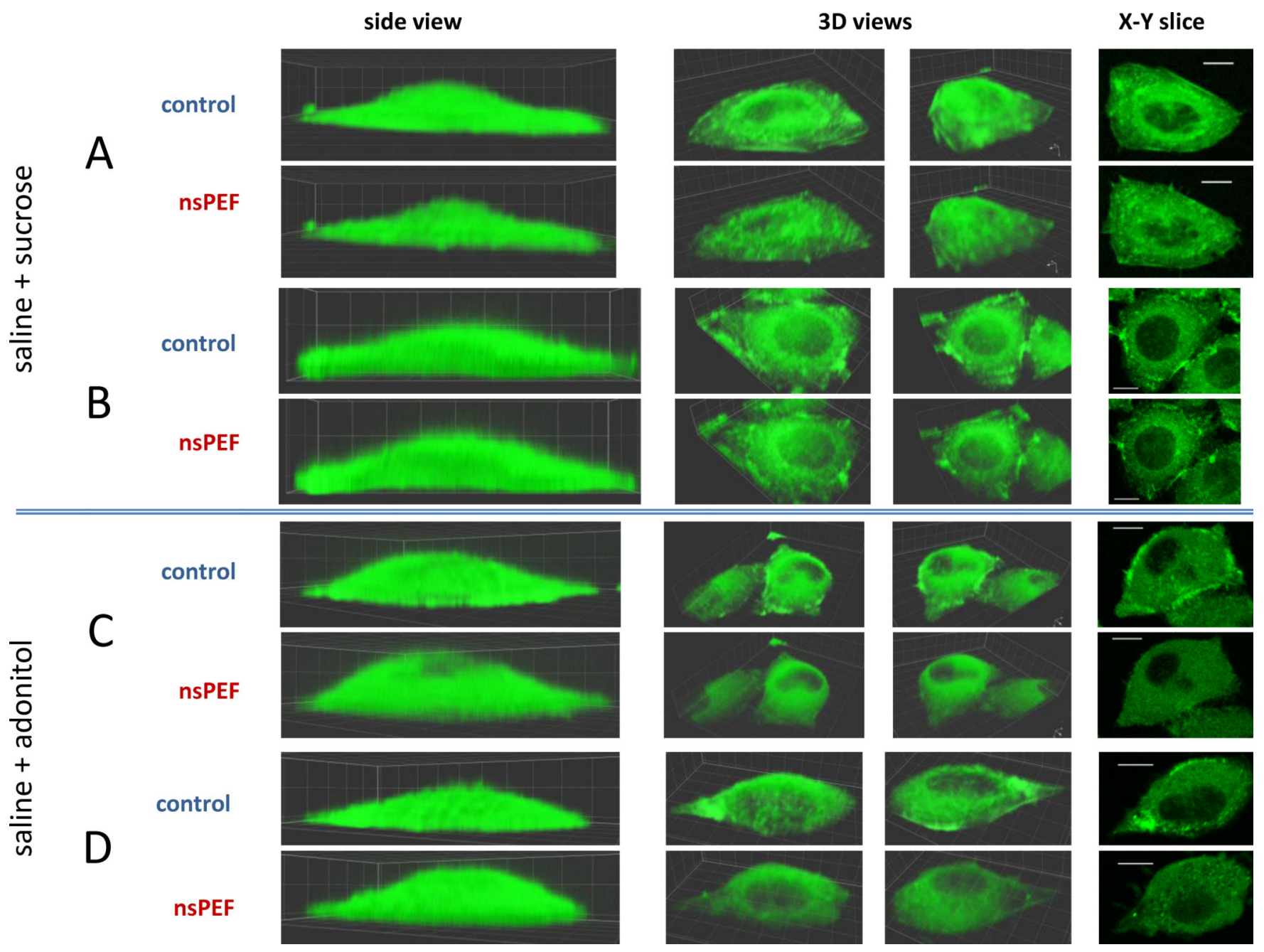

Fig. 4.

Inhibition of nsPEF-induced cell reshaping and of disassembly of actin structures by an isoosmotic addition of sucrose (A and B) but not of adonitol (C and D). A - D are four independent experiments (out of 20). In all experiments, the exposure was 4 pulses at 19.2 kV/cm (600 ns, 2 Hz). See Fig. 3 and text for other details. 


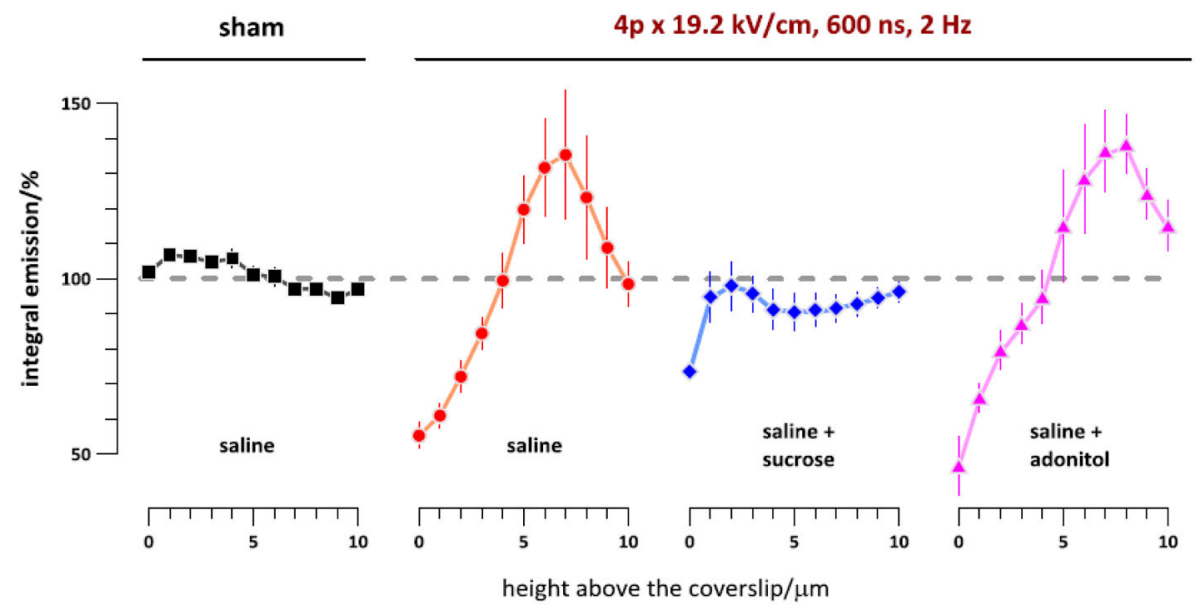

Fig. 5.

Vertical re-distribution of actin fluorescence following sham exposure (left) or nsPEF exposure in different buffers. Z-stacks of cell images were taken with 1- $\mu \mathrm{m}$ step immediately before exposure and $10 \mathrm{~min}$ after it. The buffer compositions and exposure parameters are indicated in the graph. Actin fluorescence intensity was integrated over the cell projection area in post-nsPEF images at each height and expressed in \% to the respective pre-exposure values. The data were collected from 38 individual cells. See text for more details. 


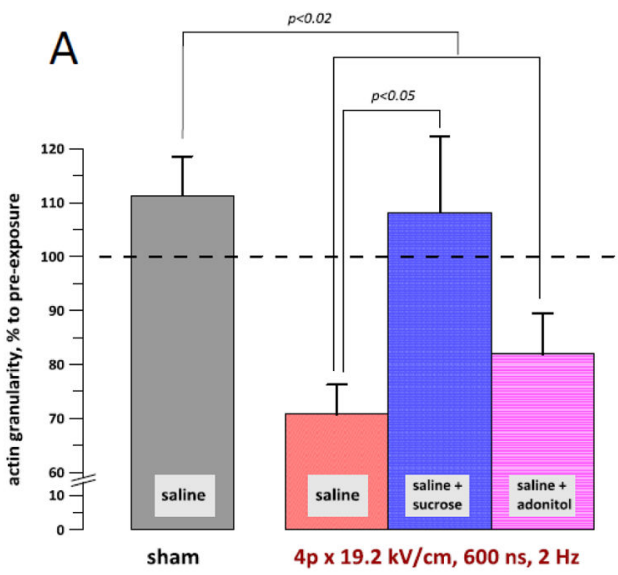

B

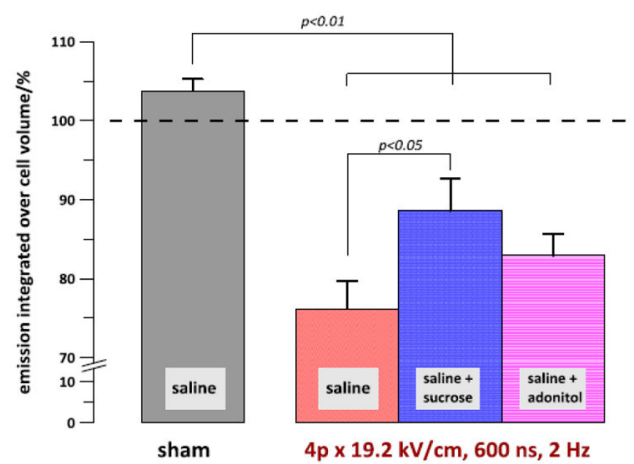

Fig. 6.

The granularity of actin (A) and the integral emission of actin within the cell volume (B) at 10 min after a sham or nsPEF exposure in different buffers. See text for details of the measurements. The data were collected from 38 individual cells. The buffer compositions and exposure parameters are indicated in the graphs. $P$ values are shown only for those groups which are statistically different. 